\title{
Tools and pipelines for BioNano data: molecule assembly pipeline and FASTA super scaffolding tool
}

\author{
Jennifer M. Shelton ${ }^{1}$, Michelle C. Coleman ${ }^{1}$, Nic Herndon ', Nanyan Lu', Ernest T. Lam², \\ Thomas Anantharaman², Palak Sheth² and Susan J. Brown ${ }^{1 *}$
}

\begin{abstract}
Background: Genome assembly remains an unsolved problem. Assembly projects face a range of hurdles that confound assembly. Thus a variety of tools and approaches are needed to improve draft genomes.

Results: We used a custom assembly workflow to optimize consensus genome map assembly, resulting in an assembly equal to the estimated length of the Tribolium castaneum genome and with an N50 of more than $1 \mathrm{Mb}$. We used this map for super scaffolding the T. castaneum sequence assembly, more than tripling its N50 with the program Stitch.

Conclusions: In this article we present software that leverages consensus genome maps assembled from extremely long single molecule maps to increase the contiguity of sequence assemblies. We report the results of applying these tools to validate and improve a 7x Sanger draft of the T. castaneum genome.
\end{abstract}

Keywords: Genome map, BioNano, Genome scaffolding, Genome validation, Genome finishing

\section{Background}

The quality and contiguity of genome assemblies, which impacts downstream analysis, varies greatly [1-3]. Initial assembly drafts, whether based on lower coverage Sanger or higher coverage next-generation sequencing (NGS) reads, are often highly fragmented. Physical maps of bacterial artificial chromosome (BAC) clones can be used to validate and scaffold sequence assemblies, but the molecular, human, and computational resources required to significantly improve a draft genome are often not available to researchers working on non-model organisms. The BioNano Irys $®$ System linearizes and images nicked and fluorescently labeled long DNA strands to generate single molecule physical maps. The Irys System provides affordable, high throughput physical maps of significantly higher contiguity with which to validate draft assemblies and extend scaffolds [4].

\footnotetext{
*Correspondence: sjbrown@ksu.edu

1 KSU/K-INBRE Bioinformatics Center, Division of Biology, Kansas State

University, Manhattan, KS, USA

Full list of author information is available at the end of the article
}

Genome assembly and scaffolding algorithms are inherently limited by the length of the DNA molecules used as starting material to generate data. Specifically, if repetitive, polymorphic or low complexity regions are longer than the single molecules used to generate data, then they cannot be resolved by bioinformatics tools with certainty. The specifications for PacBio P6-C4 chemistry [5] indicate that PacBio reads have an N50 of $14 \mathrm{~kb}$ with a maximum length of $40 \mathrm{~kb}$. Illumina Long Distance Jump Libraries can also span $40 \mathrm{~kb}$ [6]. MinION nanopore sequence reads have an average read length of $<7 \mathrm{~kb}$ [7]. Illumina TruSeq synthetic long-reads can span up to $18.5 \mathrm{~kb}$; however, they fail to assemble if the sequence has problematic regions longer than the component reads used to assemble the synthetic reads (e.g. in the heterochromatin) [8]. The OpGen Argus [9] platform produces optical maps that have a length of $150 \mathrm{~kb}$ to $2 \mathrm{Mb}$ from up to $13 \mathrm{~Gb}$ data collected per run [10]. The Irys System from BioNano Genomics produces single molecule maps that have an average length of $225 \mathrm{~kb}$ from up to $96 \mathrm{~Gb}$ data collected per run after filtering for molecules $<150 \mathrm{~kb}$ [11]. Genomic repeats can be 
much longer than the 5-40 kb that many technologies can span with a single molecule. In fact, a recent study used consensus genome maps (assembled from single molecule maps $>150 \mathrm{~kb}$ ) to identify repeats that are hundreds of $\mathrm{kb}$ in the human genome [12].

Sequence-based assembly methods are fraught with platform-specific error profiles (e.g. resolving homopolyer repeats or read-position effects on base quality) [13]. Map-based approaches offer an orthogonal genomic resource that complements sequence-based approaches but not their error profiles. For example, map-based error profiles tend to consider errors in estimated molecule or fragment length and errors associated with restriction sites that are too close together, neither of which influence sequence-based approaches $[12,14]$. Both the BioNano Irys System and OpGen Argos platform provide single molecule maps from genomic DNA. OpGen may provide higher resolution maps by using enzymes with a six rather than a seven base pair recognition site, but BioNano's single molecule maps still deliver a more efficient and affordable method for generating whole genome maps.

\section{Data formats}

The tools described make use of three file formats developed by BioNano. The Irys System images extremely long molecules of genomic DNA that are nick-labeled at seven bp motifs using one or more nicking endonucleases and fluorescently labeled nucleotides. Molecules captured in TIFF images are converted to BNX format text files that describe the detected label position for each molecule (Fig. 1(1-2)). The individual molecules described in BNX files are referred to as single molecule maps. Consensus Map (CMAP) files include the molecule map lengths and label positions for long genomic regions that are either inferred from assembly of raw single molecule maps (Fig. 1(7-8)) or in silico from sequence scaffolds (Fig. 1 (3-4)). Individual maps in these two types of CMAP files are referred to as a consensus genome map or an in silico map, respectively. The alignment between two CMAP files is stored as an XMAP file that includes alignment coordinates and an alignment confidence score (Fig. 1(10)).

\section{Other software tools for scaffolding with BioNano data}

BioNano Genomics developed the Hybrid Scaffold tool to create more contiguous consensus genome maps using information from both sequence and BioNano genome map data. These more contiguous maps can then be used to create more contiguous sequence assemblies. The Hybrid Scaffold software first creates hybrid in silico/consensus genome map contigs based on an alignment between the two. The output genome maps are called hybrid scaffolds and are aligned to the original in silico maps. This alignment is used to output a FASTA file of sequence super scaffolds. These sequences include seven base pair ambiguous-base motifs to indicate where labels occur within gaps. Because they extend into regions with consensus genome maps but without sequence data they may begin or end with gaps. The Hybrid Scaffold program only generates hybrid in silico/consensus genome map contigs, and therefore super scaffolds, if no conflicts (e.g. negative gap lengths or otherwise conflicting alignments) are indicated in the alignment of in silico and consensus genome maps. In this conservative approach, all conflicting alignments are excluded from the hybrid scaffold genome map and flagged for further evaluation at the sequence level.

\section{Motivation}

We designed tools and workflows to optimize the use of single molecule maps in the construction of whole genome maps and then use the best resultant consensus genome maps to improve contiguity of draft genome sequence assemblies. Single-molecule maps were assembled into BioNano genome maps de novo using software tools developed at BioNano [12]. As with sequence-based assembly algorithms, it was noted that testing a range of assembly parameters can improve final assembly quality for the BioNano Assembler. Additionally, applying error correction to molecule map stretch was found to improve assembly quality. Therefore, we created AssembleIrysCluster to normalize molecule map stretch and automate the writing of assembly scripts that use various parameters. We created the Stitch tool to super scaffold sequence-based assemblies using alignments to the optimal BioNano genome map. The Hybrid Scaffold and Stitch tools for genome finishing both take alignments from the BioNano RefAligner as input. Both tools were developed simultaneously but were ultimately found to be useful for distinct applications. We validated AssembleIrysCluster and Stitch using the Tribolium castaneum genome [15] because this project has genetic map resources [16] that offer independent corroboration. Genetic maps were not used as input for Stitch. In this case, the super scaffolds created by Stitch were compared to the order of scaffolds within ChLGs predicted by the genetic map.

\section{Implementation Overview}

The tools described below take raw molecule maps as input, assemble genome maps, and then use these genome maps to super scaffold draft sequence assemblies. The tool AssembleIrysCluster generates consensus genome maps for a range of assembly parameters. We developed AssembleIrysCluster to prepare BNX files for assembly and produce nine customized assembly scripts (Fig. 2c-g). Next, genome maps from the user-selected best assembly 


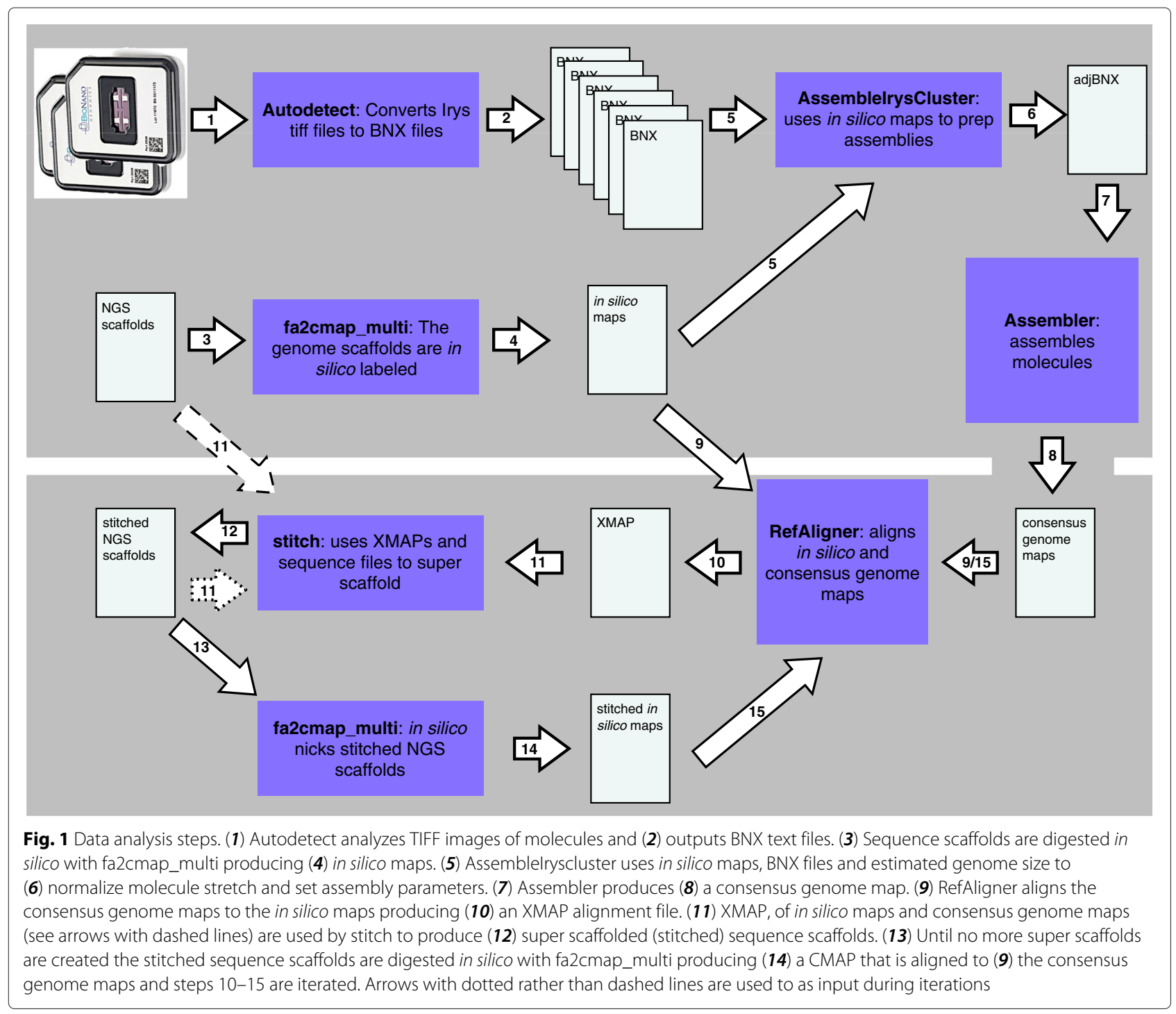

are used by the tool Stitch to validate and super scaffold sequence assemblies (Fig. 3).

\section{AssemblelrysCluster: molecule stretch}

In the first stage, AssembleIrysCluster adjusts single molecule map stretch (Fig. 2c). BioNano software operates under the assumption that imaged molecules contain 500 bases per pixel (bpp). Stretch, or bpp, can deviate from 500 bpp and this discrepancy can vary from scan to scan within a flowcell (Additional file 1). Sequence scaffolds are considered to be more accurate than raw BNX molecules in terms of label positions. Therefore molecule maps in BNX files are split by scan, and after alignment to the in silico maps, an empirical average bpp value is determined for the molecule maps in each scan. The bpp indicated by this alignment is used by RefAligner to adjust molecule map bpp to 500 . Once stretch has been evaluated and normalized, the split BNX files are merged into a single file (Fig. 2e).

We observed consistent patterns of empirically determined bpp between flowcells using the same flowcell model and chemistry when signal-to-noise ratios are optimal and the degree of genomic divergence between the samples used for the in silico maps and molecule maps are low (Additional file 1). To identify the low quality flowcells, bpp observed in alignments of scans are plotted as a quality control (QC) graph by AssembleIrysCluster (Fig. 2d).

\section{AssemblelrysCluster: customization of BioNano assembly scripts}

In the next stage, AssembleIrysCluster creates various assembly scripts to explore a range of parameter sets around the the assembler default parameters with 


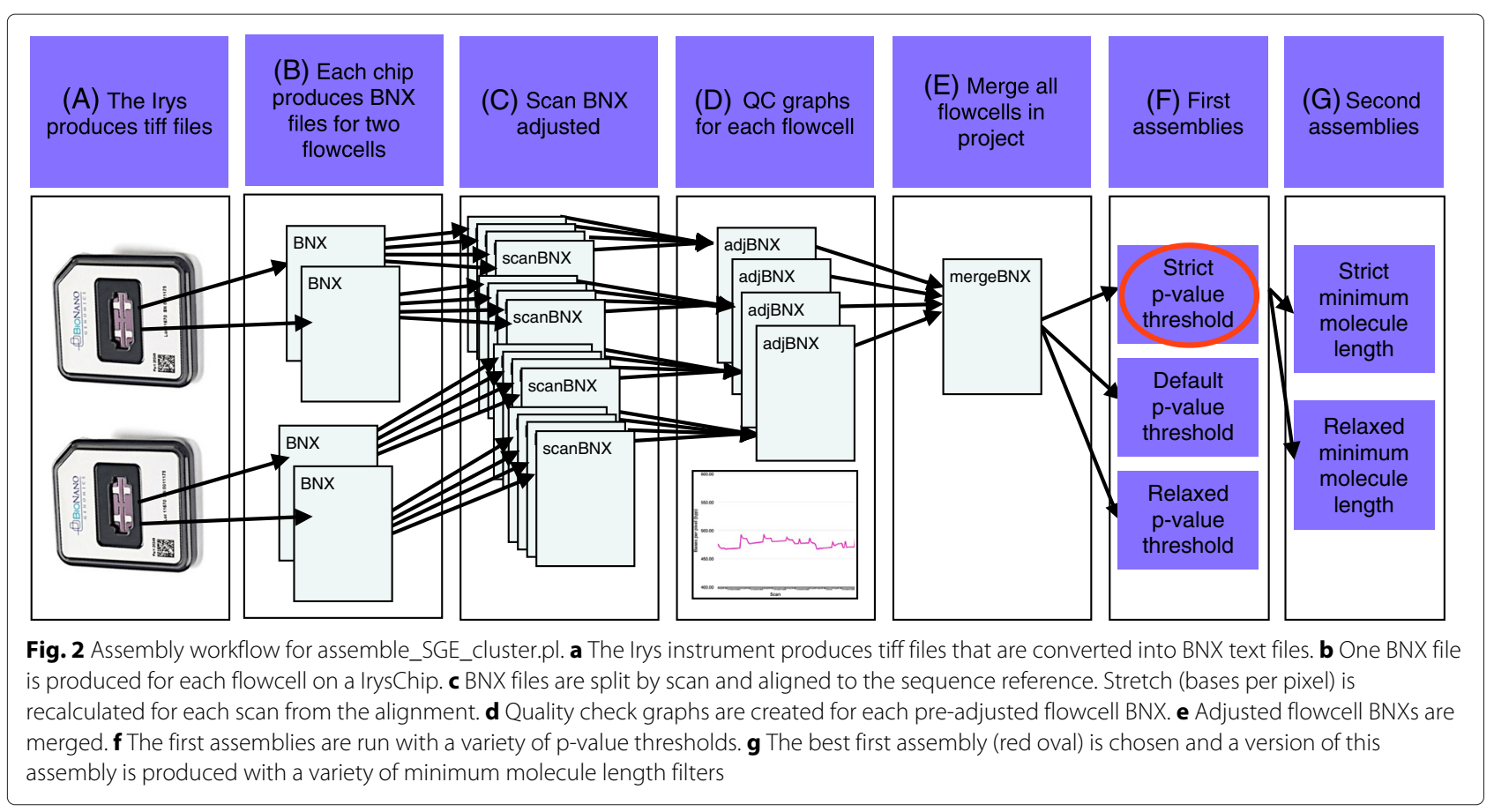

the goal of selecting the optimal assembly for downstream analysis (Fig. 2f-g). Single molecule maps in the adjusted merged BNX file are aligned to the in silico maps. An alignment error profile generated by RefAligner is used with the estimated genome length to calculate default assembly parameters; and the eight other scripts that include variants of these parameters. Initially, three assemblies are run, the first with $p$-ValueThresholdDefault $=\frac{1 e-5}{\text { GenomeLength }(M b)}$, the second with $p$-ValueThresholdStrict $=\frac{p \text {-ValueThreshold }}{10}$ and the third with $p$-ValueThresholdRelaxed = $p$-ValueThreshold $\times 10$ (Fig. 2f). The minimum molecule length is set to $150 \mathrm{~kb}$. If one of these runs does not produce a satisfactory assembly, then two minimum molecule length variants $(180$ and $100 \mathrm{~kb})$ are tested with the $p$-ValueThreshold of the current best assembly (Fig. 2g). Between three and nine assemblies are run until a satisfactory assembly is produced.

\section{BioNano assembly optimization}

The ultimate goal is to produce consensus genome maps that can be used to guide sequence-based haploid reference genome assembly. While single molecule maps can be used to reconstruct haplotypes [17], genome assembly involves collapsing polymorphisms arbitrarily into a consensus reference genome. Therefore the cumulative length of ideal consensus genome maps should equal the haploid genome length. Additionally, $100 \%$ of the consensus genome maps would align non-redundantly to $100 \%$ of the in silico maps. In practice, the best BioNano assembly is selected based on similarity to the estimated haploid genome length and minimal alignment redundancy to the reference in silico maps.

\section{Stitch: alignment filters}

For each RefAligner alignment the user designates a reference file with in silico maps from either a draft or reference quality genome or, if the user is aligning two files that both contain consensus genome maps, then a file of consensus genome maps. Additionally, the user specifies a query file with either single molecule maps or consensus genome maps.

We designed the Stitch algorithm to use alignments of query consensus genome maps to reference in silico maps in order to predict the higher order arrangement of sequence scaffolds (Fig. 3). RefAligner assumes the alignment reference has the error profile of in silico maps and the query has the error profile of consensus genome maps. Therefore alignments are run with the in silico maps as the reference, and are inverted and sorted by consensus genome map coordinates for efficient parsing by Stitch (Fig. 3a-b).

Before inferring super scaffolds from XMAP files, Stitch filters low quality alignments by confidence score. Alignments of in silico and consensus genome maps are assigned a confidence score that is the $-\log 10$ of the FalsePositivep-Value. Misaligned labels and sizing error increase the alignment FalsePositivep-Value and decrease confidence scores [18]. 


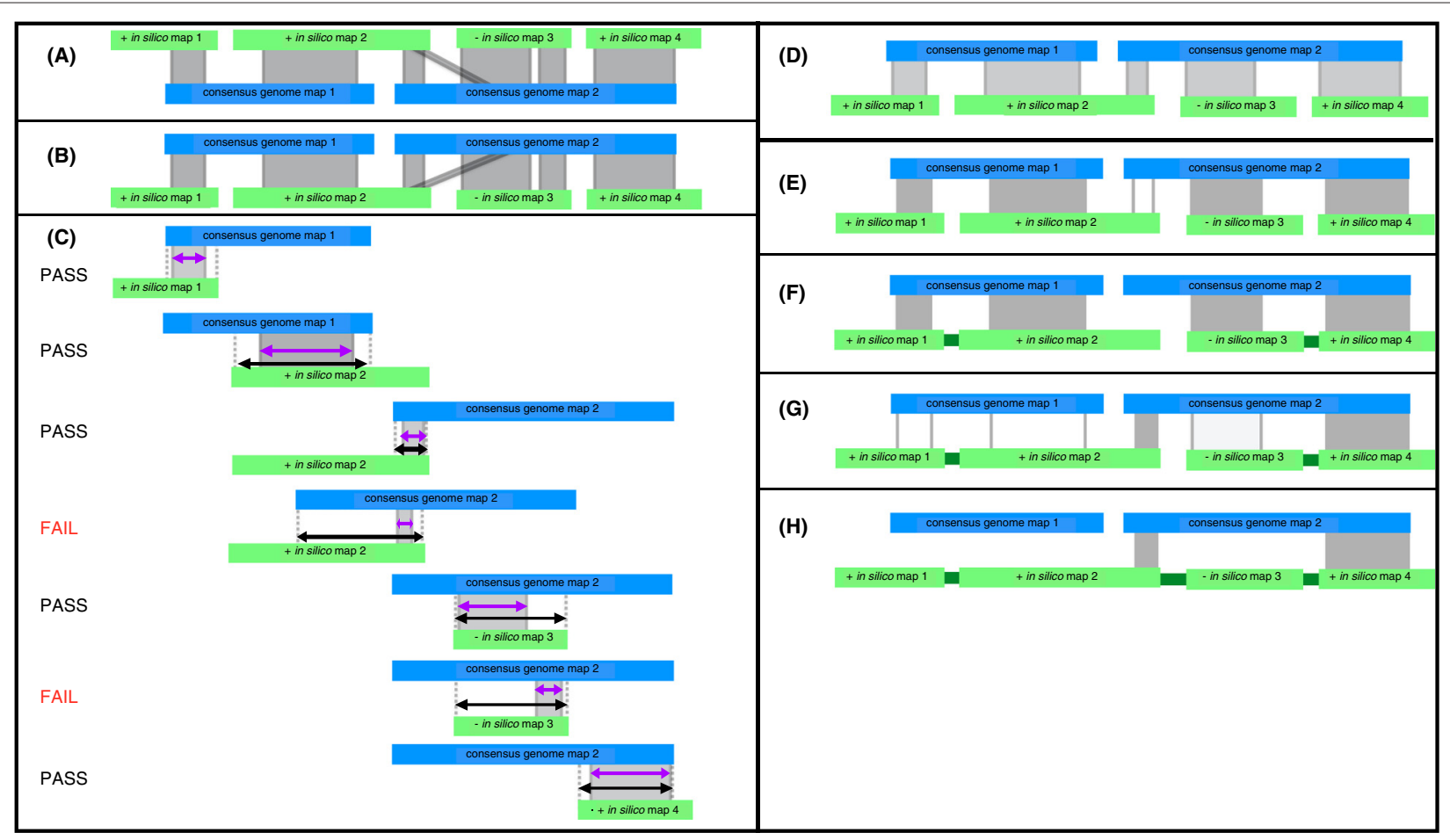

Fig. 3 Steps of the stitch.pl algorithm. Consensus genome maps (blue) are shown aligned to in silico maps (green). Alignments are indicated with grey lines. CMAP orientation for in silico maps is indicated with a " + " or "-" for positive or negative orientation respectively. a The in silico maps are used as the reference. $\mathbf{b}$ The alignment is inverted and used as input for stitch.pl. c The alignments are filtered based on alignment length (purple) relative to total possible alignment length (black) and confidence. Here assuming all alignments have high confidence scores and the minimum percent aligned is $30 \%$ two alignments fail for aligning over less than $30 \%$ of the potential alignment length for that alignment. d Filtering produces an XMAP of high quality alignments with short (local) alignments removed. e High quality scaffolding alignments are filtered for longest and highest confidence alignment for each in silico map. The third alignment (unshaded) is filtered because the second alignment is the longest alignment for in silico map 2. f Passing alignments are used to super scaffold (captured gaps indicated in dark green). $\mathbf{g}$ Stitch is iterated and additional super scaffolding alignments are found using second best scaffolding alignments. $\mathbf{h}$ Iteration takes advantage of cases where in silico maps scaffold consensus genome maps as in silico map 2 does. Stitch is run iteratively until all super scaffolding alignments are found

Super scaffolds are built from overlapping alignments. Overlapping alignments are similar to global alignments, i.e., alignments spanning from end to end for two maps of roughly equal length, but to search for overlap alignment gaps after the ends of either map are not penalized. The RefAligner scoring scheme does not currently have a parameter to favor overlapping alignments, e.g., to initialize the dynamic programming matrix with no penalties and take the maximum score of the final row or column in the matrix. RefAligner reports local alignments between two maps and applies a fixed penalty based on the user-defined likelihood of unaligned labels at the ends of the alignment. Raising or lowering this penalty selects for local or global alignments, respectively, but neither option favors overlapping alignments specifically. Stitch filters by the percent of the total possible alignment length that is aligned (Fig. 3c). To approximate scoring that favors overlapping alignments, Stitch uses thresholds for minimum percent of total possible aligned length, the percent aligned threshold (PAT).

Similar to scoring structures that favor overlapping alignments, PAT filters out local alignments. However, unlike a scoring structure, PAT is applied after alignment and therefore cannot result in the aligner exploring possible extensions into an overlap but instead favors a shorter local alignment with a higher cumulative score. Therefore Stitch accepts alignments with less than $100 \%$ PAT. Default values for the PAT were determined empirically after reviewing the degree to which filtered alignments agreed with the independently derived genetic maps of T. castaneum and by visual inspection of alignments.

In practice we used two sets of alignment filters and kept alignments that passed one or both sets. The first set had a low PAT and a high confidence score threshold. The second set had a higher PAT and a lower confidence score and was intended to identify longer overlaps especially in regions of the genome where label density is low. 


\section{Stitch: super scaffolding}

Scaffolding alignments are selected from the remaining high quality alignments (i.e., more than one in silico map aligns to the same consensus genome map (Fig. 3d)). For each in silico map with more than one high quality, scaffolding alignment the longest alignment for the in silico map is selected (Fig. 3d-e). If alignment length is identical then the highest confidence alignment is selected. If confidence scores are identical then an alignment is chosen arbitrarily.

Gap lengths between in silico maps are inferred from scaffolding alignments and used to create new super scaffolds (Fig. 3f) in a new genome FASTA file and associated AGP file. If gap lengths are estimated to be negative, Stitch adds a $100 \mathrm{bp}$ spacer gap to the sequence file and indicates that the gap is type "U" for unknown in the AGP.

Stitch only makes use of one alignment per in silico map each iteration. Stitch can be run iteratively (Fig. 1(10-15)) such that each successive output FASTA file is converted into in silico maps and aligned to the original consensus genome maps. This alignment is inverted and used as input for the next iteration. Subsequent iterations of Stitch will make use of any in silico maps that join growing super scaffolds, effectively using both sequence data and genome maps to stitch together the final super scaffolds (Fig. 3g-h).

\section{Stitch: flagging potential Mis-assemblies}

This algorithm is meant to be an intermediate refinement of draft genomes prior to further fine scale refinement at the sequence level. Inconsistencies between the consensus genome maps and the in silico maps are reported in output logs to facilitate downstream sequence editing. If an alignment passes initial confidence score and PAT filtering but has a PAT less than $60 \%$, this is reported as a partial alignment. A partial alignment may occur if either the sequence scaffold or the consensus genome map is the result of a chimeric or erroneous assembly. Additionally, if a gap length is estimated to be negative, it may indicate that the sequence scaffolds can be joined with a local assembly or that an incorrect assembly needs to be broken within a scaffold. Assembly errors in the consensus genome maps or spurious alignments could also result in either of these cases. Ideally researchers could make use of the alignment of genomic sequence reads to the genome sequence assembly and the alignment of single molecule maps to the consensus genome maps to determine which assembly is likely to be incorrect.

\section{Post analysis: software updates}

Since completion of the $T$. castaneum genome update we have released updates for both Stitch and AssembleIrysCluster. Stitch (version 1.4.5) now allows the user to set a minimum negative gap length filter for alignments. In the event that two in silico maps have a negative gap length smaller than this value, which is equivalent to a longer overlap of the sequence scaffolds, Stitch will automatically exclude both in silico maps from consideration when super scaffolding. This new feature allows users to further customize the stringency of the Stitch output. In addition, BioNano Genomics has updated Assembler to automate per-scan stretch adjustment. Finally the KSU K-INBRE Bioinformatics Core has moved to producing assemblies on a Xeon Phi server with 1488 threads $(6 \times 60 \times 4$ Xeon Phi co-processor threads $+24 \times 2$ Xeon host threads) and 256 GB of host RAM $+6 \times 8$ GB Xeon Phi Ram, and Linux CentOS 7 operating system. Because of all of these changes we opted to create a new assembly workflow rather than update AssembleIrysCluster. The most current assembly workflow, AssembleIrysXeonPhi maintains all the functionality of AssembleIrysCluster (e.g. adjusting stretch by scan and writing assembly scripts with all combinations of three $p$-ValueThresholds and three Minlen parameters) but runs on our new machine with the latest release of the BioNano Assembler and RefAligner.

\section{Post analysis: tutorials and complete pipelines}

Additionally, since completion of the $T$. castaneum genome update we have created three pipelines for BioNano data complete with sample datasets and tutorials. The Sewing machine pipeline iteratively super scaffolds genome FASTA files with BioNano genome maps using stitch.pl and the BioNano tool RefAligner until no new super scaffolds can be produced. The pipeline runs alignments with both default and relaxed parameters. These alignments are then used by stitch.pl to superscaffold a fragmented genome FASTA. The "Raw data-to-finished assembly and assembly analysis" pipeline for BioNano molecule maps with a sequence-based genome FASTA prepares raw molecule maps and writes and runs a series of assemblies for them. Then the user selects the best assembly and uses this to super scaffold the reference FASTA genome file and summarize the final assembly metrics and alignments. The "Raw data-to-finished de novo assembly and assembly analysis" pipeline for BioNano molecule maps is for de novo projects. Both of the last two pipelines are broken into stages at points were the user must select the best set of parameters from assemblies or alignments that have been run by the pipeline.

\section{Results and discussion \\ Dataset generation}

High molecular weight DNA was isolated from young Tribolium castaneum pupae from the GA2 line that was inbred 20 generations. The GA2 line was also used for the 
genome sequence-based assembly. Ethical approval was not sought for the study because the study organism is an insect.

Using Knickers (BioNano Genomics), an in silico label density calculator, we estimated that the Tribolium castaneum genome had 8.66 and 5.51 labels per $100 \mathrm{~kb}$ for the nt.BspQI and nt.BbvCI enzymes (New England BioLabs) respectively. The ideal number of labels per $100 \mathrm{~kb}$ is between 10 and 15 therefore we nicked with both enzymes. DNA was nicked, labeled with fluorescent nucleotides, and repaired according to BioNano protocol; and 93 BNX files were produced from the Irys genome mapping system (Fig. 4 and Additional file 2). Four corrupted files (cumulative length $=0$ ) were excluded from this analysis. All T. castaneum BNX files have been deposited to labarchives (doi:10.6070/H4V69GK3).

The high number of BNX files produced is due to several factors. Typically one BNX file is produced per flowcell; however, in certain cases after the initial number of scans, additional scans were run producing an additional BNX file. Another reason for the large number of files is that data was originally generated using the
IrysChip $\mathbb{R}$ V1 while the Irys System was under beta testing. Over time, maximum cumulative length per BNX file increased (Fig. 4 and Additional file 2). After the upgrade to the IrysChip V2, an increase was also observed in maximum data per BNX file (Fig. 4 and Additional file 2).

Molecule map quality metrics were calculated using bnx_stats (version 1.0). We generated $\sim 250 \mathrm{x}$ coverage of the $T$. castaneum genome for single molecule maps $>150 \mathrm{~kb}$, the default minimum molecule map length. The 239,558 single molecule maps with lengths $>150 \mathrm{~kb}$ had an N50 of $202.63 \mathrm{~kb}$ and a cumulative length of $50.6 \mathrm{~Gb}$ (Table 1). Histograms of per-molecule quality metrics for maps after applying a minimum length filter of either 100, 150 and $180 \mathrm{~kb}$ are reported in Additional file 3.

To generate the in silico maps, we used the sequencebased assembly scaffolds from version 5.0 of the Tribolium castaneum genome (Tcas5.0). Version 5.0 (Table 2) of the $T$. castaneum genome is an updated version of the sequence assembly that was created by adding $1.03 \mathrm{Mb}$ of sequence to version 3.0 [15]. Two hundred and twentythree of the 2240 scaffolds within Tcas 5.0 were longer than $20 \mathrm{~kb}$ with more than 5 labels, the minimum requirements

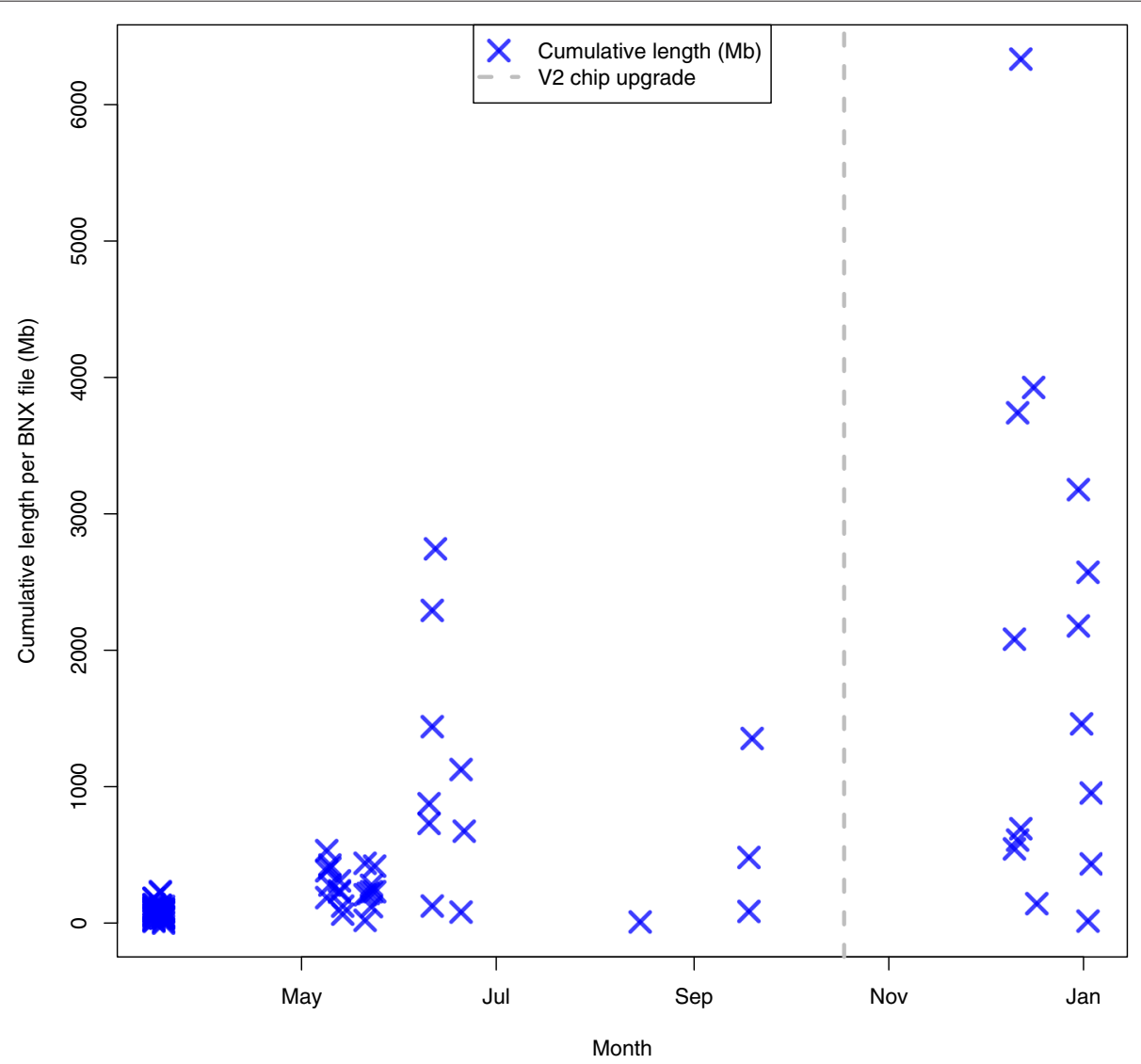

Fig. 4 Cumulative length per BNX file for T. castaneum data generated over time. Cumulative length of single molecule maps $>150 \mathrm{~kb}$ are plotted on the $y$-axis (purple X), the upgrade to the V2 IrysChip (grey dashed line) is plotted and date is indicated on the $x$-axis. Data was generated from 03-2013 to 01-2014. Aborted runs (cumulative length $=0$ ) excluded 
Table 1 Single molecule maps from T. castaneum filtered by minimum length. Molecule map N50, cumulative length and number of maps are listed for all three molecule length filters for the T. castaneum genome data

\begin{tabular}{llll}
\hline $\begin{array}{l}\text { Minimum molecule } \\
\text { map length }(\mathrm{kb})\end{array}$ & $\begin{array}{l}\text { Molecule map } \\
\text { N50 }(\mathrm{kb})\end{array}$ & $\begin{array}{l}\text { Cumulative } \\
\text { length }(\mathrm{Mb})\end{array}$ & $\begin{array}{l}\text { Number of } \\
\text { molecule maps }\end{array}$ \\
\hline 100 & 165.35 & $82,738.71$ & 503,414 \\
150 & 202.64 & $50,579.12$ & 239,558 \\
180 & 232.57 & 34287.15 & 139,949 \\
\hline
\end{tabular}

for an in silico map. These longer sequence scaffolds represent the bulk of the sequence assembly, 152.53 of the 160.74 Mb (Table 2).

\section{Assembly: selecting the optimal BioNano assembly}

Single molecule maps were assembled de novo into five distinct BioNano genome maps for T. castaneum.

Molecule maps were prepared for assembly (Fig. 2c) and noise parameters were estimated (Fig. 2d-e) using AssembleIrysCluster (version 1.0). Specifically, AssembleIrysCluster calculated three "-T" parameters (5e-10, 5e-09 and 5e-08) based on the estimated genome size, and adjusted molecule map stretch and estimated molecule map noise parameters based on alignment to in silico maps. Assemblies with these p-value thresholds are named Relaxed-T, Default-T and Strict- $T$, respectively.

In the first round of selection, the Strict- $T$ assembly was the best of these three assemblies because it has a cumulative size close to $200 \mathrm{Mb}$ (Table 2 and Fig. 5), the estimated size of the T. castaneum genome, and a small difference between non-redundant aligned length or breadth of alignment, and total aligned length (Fig. 5, Table 3 and Additional file 4). Thus in the second round of selection, Strict-T parameter was used for two further assemblies that had relaxed minimum

Table 2 T. castaneum assembly summary. Assembly metrics for Tcas5.0 (the starting sequence scaffolds), the Tcas5.0 in silico maps, the consensus genome map of assembled molecule maps, the automated output of Stitch (Tcas5.1), the manually curated sequence assembly (Tcas5.2) and the sequence assembly produced by the BioNano Hybrid Scaffold software for the T. castaneum genome

\begin{tabular}{llll}
\hline & N50 (Mb) & Number & $\begin{array}{l}\text { Cumulative } \\
\text { Length (Mb) }\end{array}$ \\
\hline Tcas5.0 sequence scaffolds & 1.16 & 2240 & 160.74 \\
Tcas5.0 in silico maps & 1.20 & 223 & 152.53 \\
Consensus genome maps & 1.35 & 216 & 200.47 \\
Tcas5.1 sequence scaffolds & 3.85 & 2148 & 165.72 \\
Tcas5.2 sequence scaffolds & 4.46 & 2150 & 165.92 \\
Tcas BioNano hybrid scaffolds & 1.83 & 2210 & 175.54 \\
\hline
\end{tabular}

molecule length (Relaxed-Minlen) of $100 \mathrm{~kb}$ rather than the $150 \mathrm{~kb}$ default or a strict minimum molecule length (Strict-Minlen) of $180 \mathrm{~kb}$. The Strict-TAndStrict-Minlen assembly improved alignments by reducing redundancy slightly. However the cumulative length of the assembly was $21.45 \mathrm{Mb}$ smaller than the estimated genome size. The Strict-TAndRelaxed-Minlen assembly had a worse cumulative length and alignment redundancy than the Strict-T assembly. Because neither of the assemblies using 100 or $180 \mathrm{~kb}$ as the minimum molecule length improved both assembly metrics when compared to the Strict-T assembly, generated in the first round of selection with the default Minlen of $150 \mathrm{~kb}$, the Strict-T assembly will be referred to as the $T$. castaneum consensus genome maps in further analysis.

The $T$. castaneum consensus genome maps have an $\mathrm{N} 50$ of $1.35 \mathrm{Mb}$, a cumulative length of $200.47 \mathrm{Mb}$, and 216 genome maps (Table 2 and Fig. 5). T. castaneum consensus genome maps aligned to $124.04 \mathrm{Mb}$ of the in silico maps created from the Tcas 5.0 assembly validating $81 \%$ of the draft sequence assembly (Table 3 and Fig. 5). Assembly metrics were calculated using the BNGCompare script (version 1.0). More detailed assembly metrics for all five assembled consensus genome maps are available in Additional file 4. Assembled T. castaneum genome maps have been deposited to labarchives (doi: $10.6070 / \mathrm{h} 42 \mathrm{f} 7 \mathrm{kf} 2$ ).

\section{Stitch: automated and manually edited assemblies}

Tcas5.1 is the output of Stitch (version 1.4.4) run for five iterations with two sets of alignment filters. To select quality alignments from regions of high and low label density, the first minimum confidence was 13 and the $P A T$ was 30 and the second minimum confidence was 8 and the $P A T$ was 90 . The resulting super scaffolds showed a greater than three-fold increase in N50 from 1.16 to $3.85 \mathrm{Mb}$ (Table 2).

The Tcas5.1 super scaffolds captured an additional 92 gaps between Tcas5.0 sequence scaffolds. Sixty-six gaps were estimated to have positive gap lengths and were represented in the sequence assembly with their estimated size (Fig. 6). Twenty-six gaps were estimated to have negative lengths and were represented with spacers of 100 N's (Fig. 6). Extremely small negative gap lengths $(<-20 \mathrm{~kb})$ were flagged for further evaluation at a sequence level. In some cases, extremely small negative gaps lengths suggest that a chimeric sequence scaffold may need to be broken at the sequence level and its fragments incorporated into different chromosome linkage groups (ChLGs). For example, half of scaffold 81 from Tcas5.0 aligns between scaffolds 80 and 82 on ChLG5 (Fig. 7a) while the other half aligns between scaffolds 102 and 103 from ChLG7 (Fig. 7b). Scaffold 81 from Tcas5.0 was placed in ChLG5 in the T. castaneum genetic 


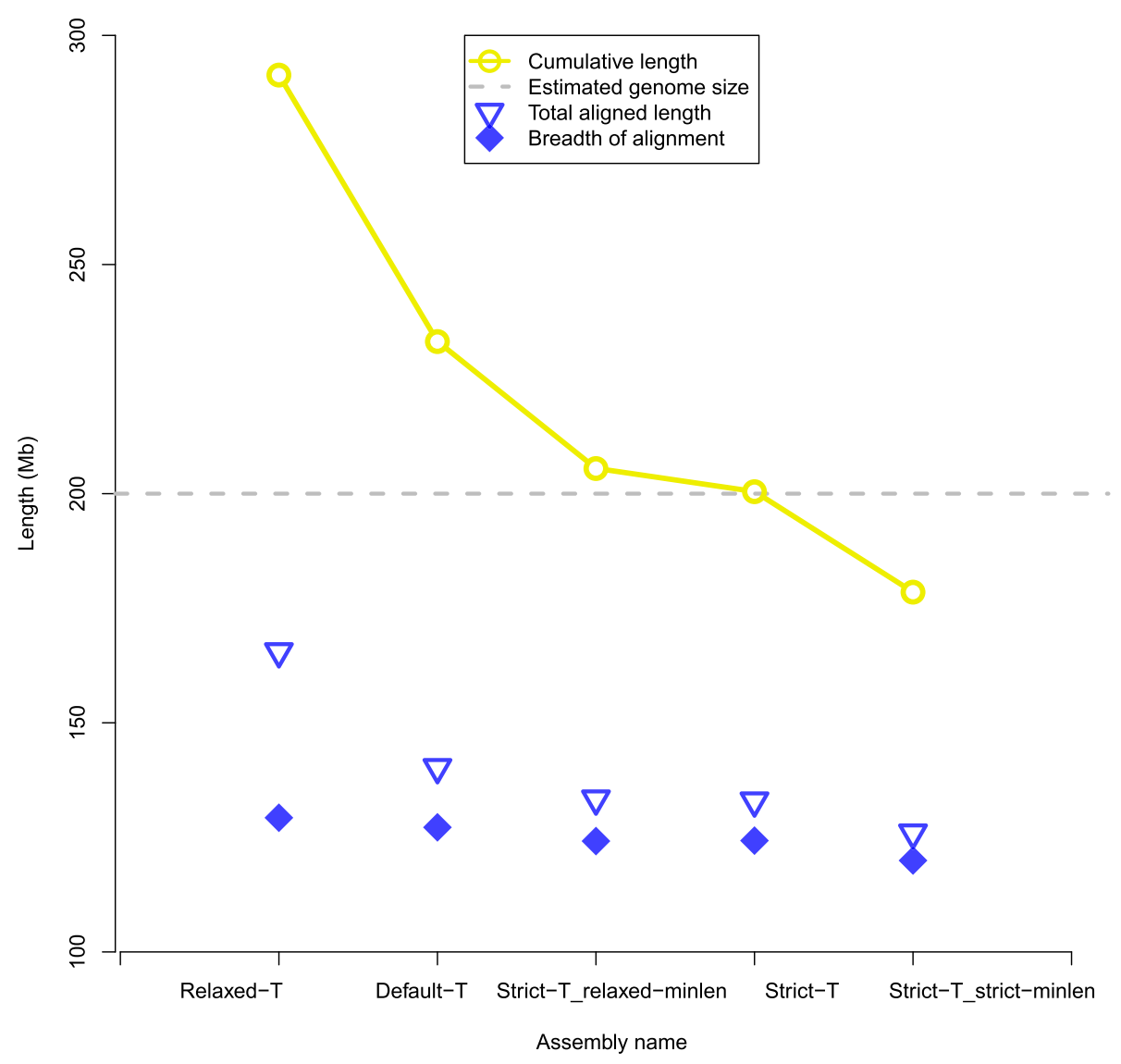

Fig. 5 Comparison of assemblies from the T. castaneum single molecule maps using five sets of parameters. Relaxed, default and strict "-T" parameters were set to 5e-08, 5e-09 and 5e-10. Relaxed, default and strict minimum molecule length were set to 100, 150 and $180 \mathrm{~kb}$

map. The arrangement supported by the genetic map was selected for cases like this where manual editing was required. The manually curated assembly is referred to as Tcas5.2. In Tcas5.2, joins were also manually accepted if they agreed with the genetic map but the alignment quality was low. Ultimately, Tcas5.2 had a higher N50 than the automated Stitch output, $4.46 \mathrm{Mb}$ (Table 2), with 66 gaps with positive estimated lengths and 24 negative length gaps.

Nearly every ChLG was less fragmented in the Tcas5.2 assembly than in the Tcas5.0 assembly. The number of scaffolds was reduced for each ChLG (Table 4) except for the 26 relatively short $(\mathrm{N} 50=0.05 \mathrm{Mb})$ and unlocalized scaffolds from ChLGY. For example, ChLGX was reduced from 13 scaffolds (Fig. 8b) to 2 in the final super scaffold or chromosome build (Fig. 8a). Five scaffolds were reoriented in ChLGX based on alignment to the consensus genome maps (Fig. 8a-b). Scaffolds were also reordered based on alignment to consensus genome maps. For example, scaffold 2 from ChLGX aligned between scaffold 36 and 37 of ChLG 3 and was therefore moved in Tcas5.2 (Additional file 5). Also, 19 previously unplaced scaffolds were anchored within a ChLG (Table 4). Improvements in Tcas 5.2 over Tcas5.0 are shown in alignments of the respective in silico maps to consensus genome maps for all ChLGs in Additional file 5.

Table 3 Alignment of T. castaneum consensus genome maps to the in silico maps of Tcas5.0. Breadth of alignment coverage (nonredundant alignment), length of total alignment (including redundant alignments) and percent of CMAP covered (non-redundantly) were calculated for the in silico maps and the consensus genome maps of the T. castaneum genome the using xmap_stats.pl

\begin{tabular}{llll}
\hline & Breadth of alignment coverage $(\mathrm{Mb})$ & Length of total alignment $(\mathrm{Mb})$ & Percent of CMAP aligned \\
\hline Tcas5.0 in silico maps & 124.04 & 132.40 & 81 \\
Consensus genome maps & 131.64 & 132.34 & 67 \\
\hline
\end{tabular}




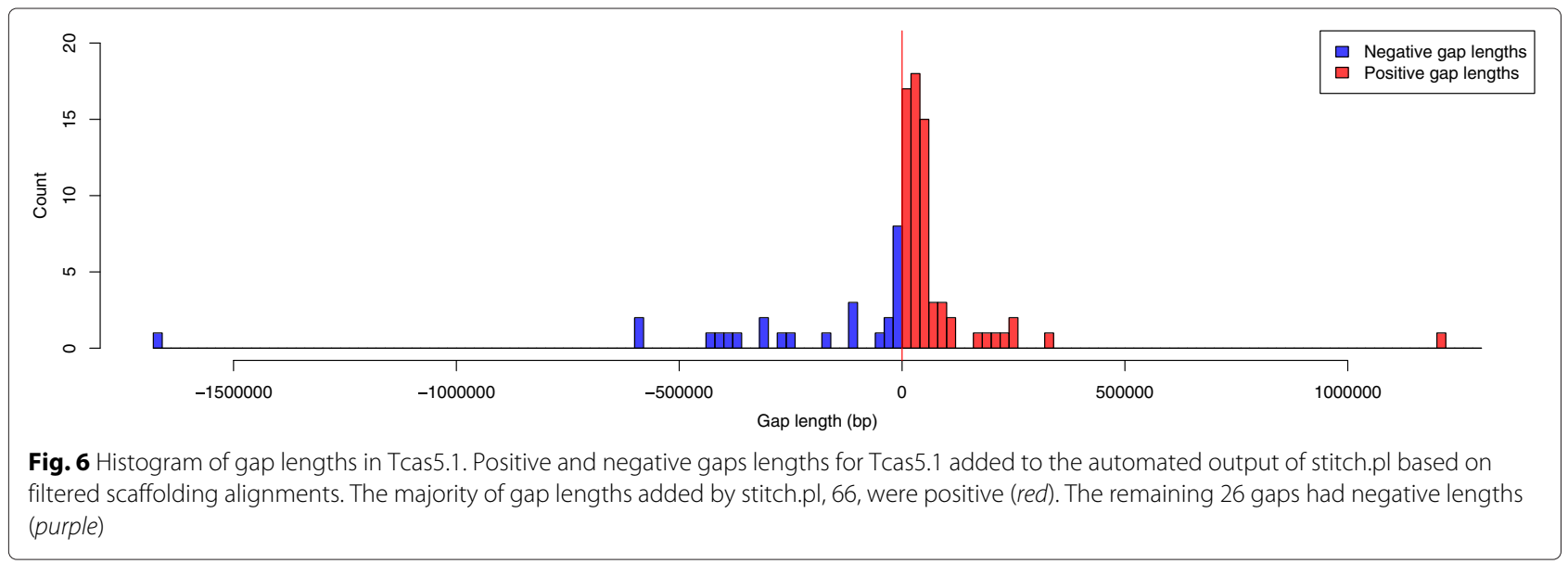

The Tcas5.2 Whole Genome Shotgun project has been deposited at DDBJ/EMBL/GenBank under the accession AAJJ00000000. The version described in this paper is version [DDBJ:AAJJ02000000,EMBL:AAJJ02000000, GenBank:AAJJ02000000]. Two scaffolds were removed from the genome assembly because they were identified as contaminants after they blasted to the Bos frontalis genome.

\section{Assembly: putative Haplotypes}

Evidence of putative haplotypes was found during visual inspection of alignments. Although overall alignment redundancy was rare for the Strict- $T$ assembly; when observed, it usually consisted of only two consensus genome maps aligning to the same in silico map (Fig. 9). This redundancy might indicate collapsed repeats in the sequence-based assembly. On the other hand it could also indicate segmental duplications, assembly of alternative haplotypes, or mis-assembly producing redundant consensus genome maps. In Fig. 9a, two consensus genome maps aligned to the same in silico map. One consensus genome map aligned across most of the in silico map while only a small region of the other consensus genome maps aligned to the the in silico map (Fig. 9a). Molecule map coverage decreased for each consensus genome map in the region where only one of them aligned to the in silico map (Fig. 9a-c). Taken together, the region of lower coverage and the number of consensus genome

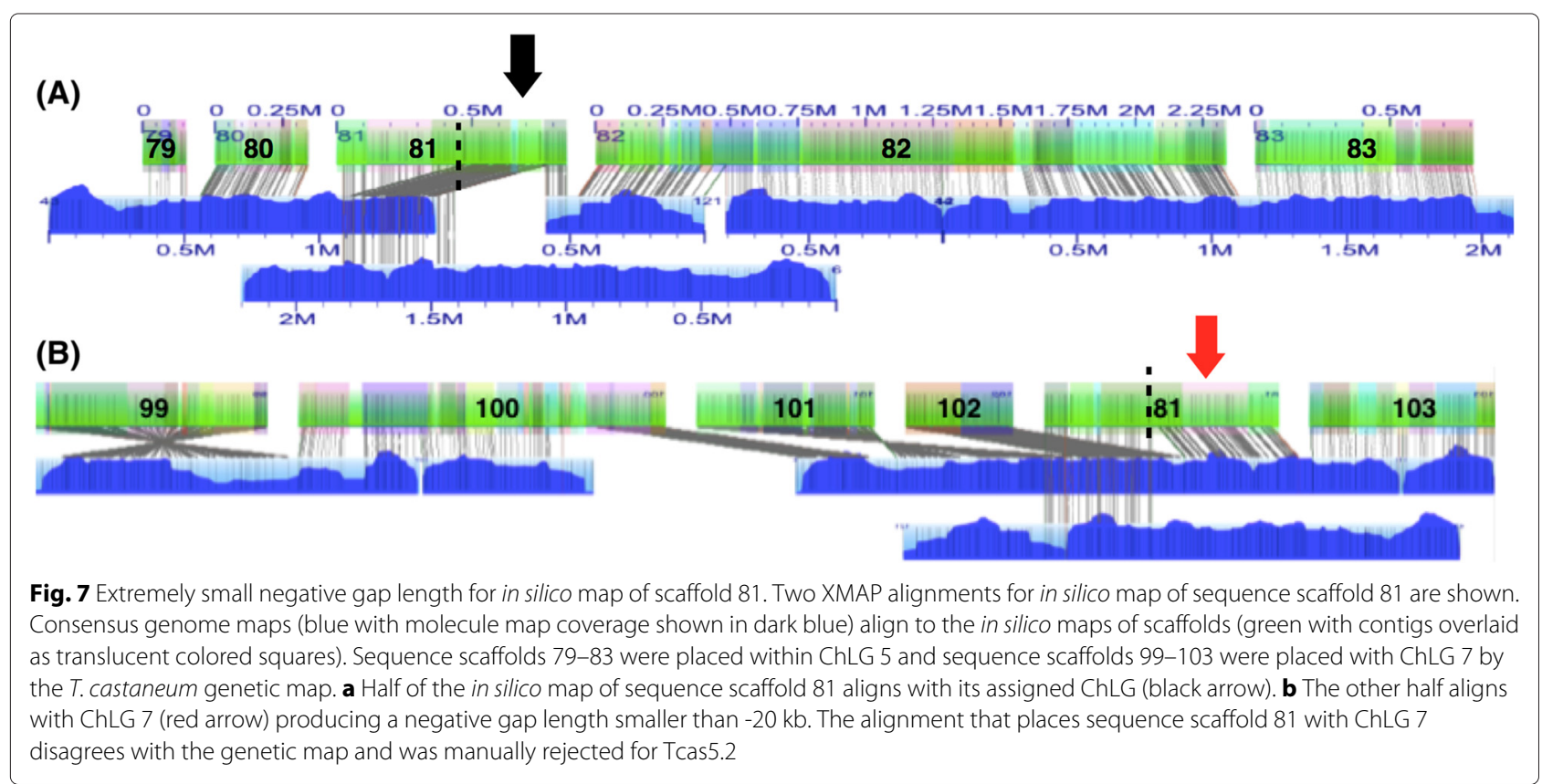


Table 4 Each T. castaneum chromosome linkage group (ChLG) before and after super scaffolding. The number of sequence scaffolds in the ordered Tcas5.0 ChLG bins and the number of sequence super scaffolds and scaffolds in the Tcas5.2 ChLG bins. The number of sequence scaffolds that were unplaced in Tcas 5.0 and placed with a ChLG in Tcas5.2 is also listed

\begin{tabular}{llll}
\hline ChLG & Tcas5.0 scaffolds & $\begin{array}{l}\text { Unplaced scaffolds } \\
\text { added in Tcas5.2 }\end{array}$ & $\begin{array}{l}\text { Tcas5.2 super } \\
\text { scaffolds }\end{array}$ \\
\hline$X$ & 13 & +2 & 2 \\
2 & 18 & +1 & 10 \\
3 & 29 & +4 & 20 \\
4 & 6 & +2 & 2 \\
5 & 17 & +1 & 4 \\
6 & 12 & +6 & 6 \\
7 & 15 & - & 6 \\
8 & 14 & +1 & 8 \\
9 & 21 & - & 9 \\
10 & 12 & +2 & 10 \\
Total & 157 & 19 & 77 \\
\hline
\end{tabular}

maps aligning (two) may indicate the assembly of two haplotypes.

\section{Stitch: comparison to other software}

We also ran Hybrid Scaffold (BioNano version 3076) to improve the $T$. castaneum sequence assembly using the T. castaneum consensus genome maps and the in silico maps from Tcas5.0. Although Hybrid Scaffold improved the scaffold N50 of the sequence-based assembly from
1.16 to $1.83 \mathrm{Mb}$, the increase in N50 was not as great as the increase after running Stitch $(\mathrm{N} 50=3.85 \mathrm{Mb})$ (Table 2). The total number of scaffolds in the sequence assembly decreased by 30 after running Hybrid Scaffold; in comparison, Stitch reduced the number of scaffolds by 92 . Stitch increased the length of the assembly by $4.98 \mathrm{Mb}$ while Hybrid Scaffold increased assembly length by 14.80 Mb. The additional increase in length from Hybrid Scaffold is likely due to extension of sequence-based scaffolds with end gaps introduced from genome map contigs that overlap the start or end of a sequence-based scaffold.

Overall, we found Hybrid Scaffold to be more conservative than Stitch. For example in Fig. 8b, the alignment of 13 in silico maps to three consensus genome maps was input into both Stitch and Hybrid Scaffold. In this alignment, the order of 11 of these in silico maps agreed with the order suggested by the genetic map (after reorienting three in silico maps). The in silico maps 12 and 13 aligned with a negative gap length between them, suggesting they may be mis-assembled or that local assembly may collapse the assembly in this region. There are several possible approaches when considering this kind of conflicting evidence. The approach of Stitch was to record that we have confirmed the relative position of these scaffolds in the larger context of the genome by creating a new super scaffold containing 100 bp spacer gaps to indicate that exact overlap or gap length is unclear (Fig. 8a). Stitch also reports the negative gap length to indicate the need for further sequence level evaluation at a later date. Alternatively, Hybrid Scaffold only automates

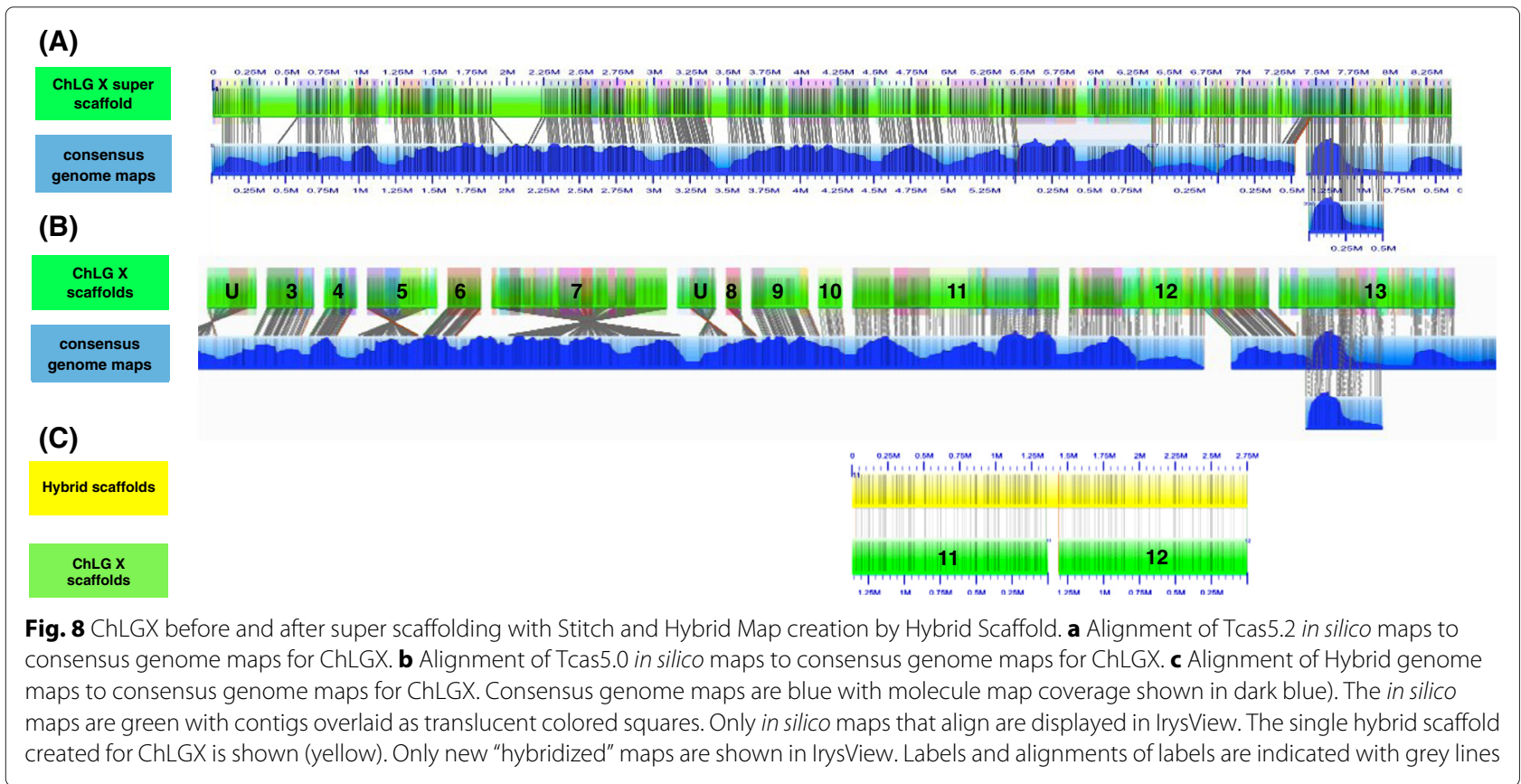




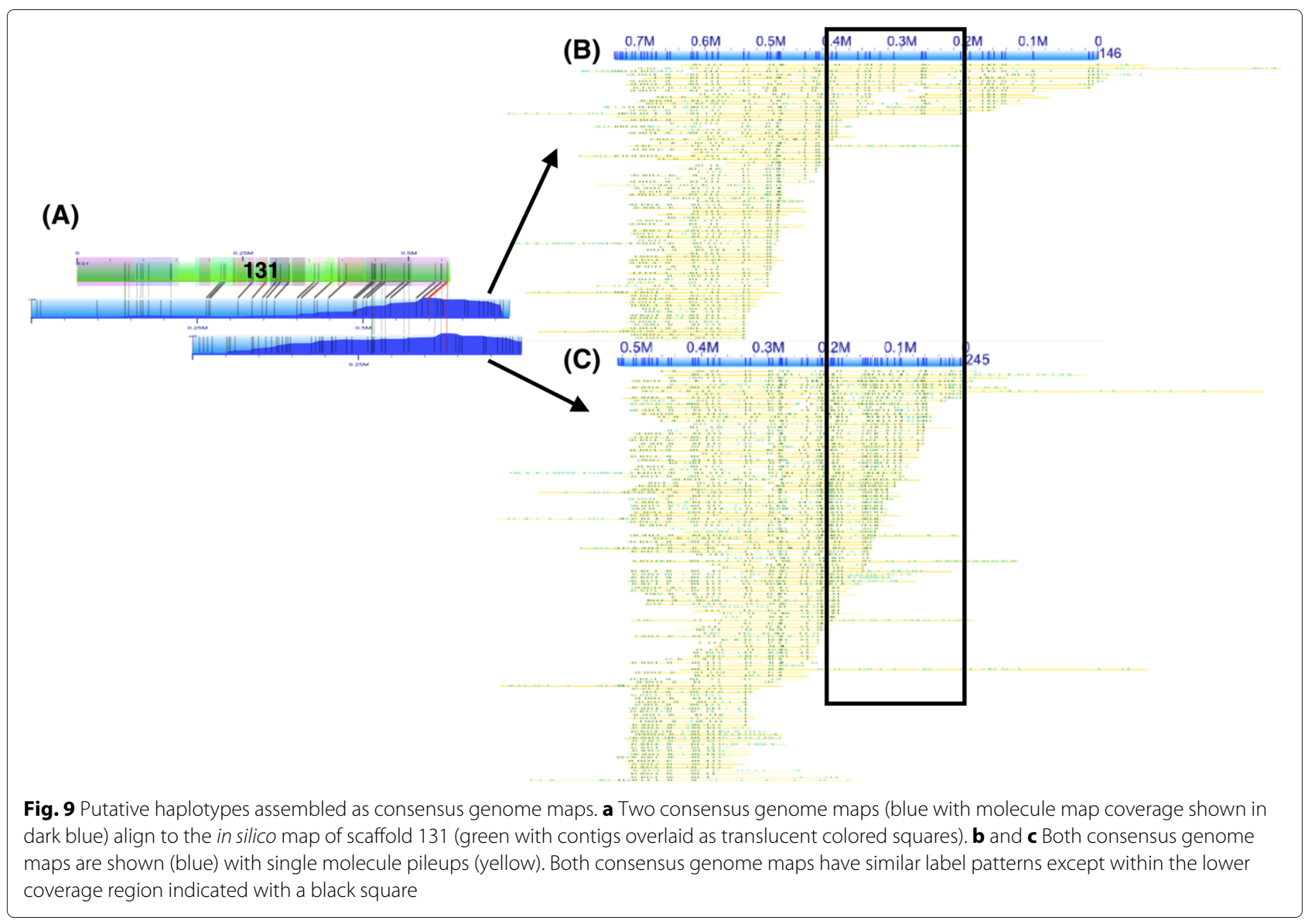

genome improvements that are unambiguously supported by all lines of evidence (sequence-based and genome mapbased) and leaves any ambiguous decisions to a human curator. Therefore, Hybrid Scaffold would only report the conflicting alignment. This is why scaffold 13, for example, is not included in the hybrid map produced by Hybrid Scaffold (Fig. 8c).

For highly refined genome assemblies, this lack of tolerance for noisy alignments has not hindered improvement of genome projects. However, for less refined genome assemblies this may be too stringent. The Hybrid Scaffold software, for example, was developed to scaffold the human genome and has been found to work well for this application. Genome projects at earlier stages would benefit from staged release of updates (e.g., immediate release of general improvement in scaffold order and orientation followed by further refinement at the sequence level). For projects such as $T$. castaneum, a more aggressive algorithm such as Stitch may be preferred in order to release the bulk of the new information about the higher order arrangement of the genome to the community of $T$. castaneum researchers quickly. Further refinement at a sequence level can be released in subsequent genome updates as they are completed.

\section{Stitch: assembly and super scaffolding with multiple genera}

We examined experiments from 16 different genera to determine if the results seen for the Tribolium castaneum genome are typical for other genomes as well. The T. castaneum genome map N50 was found to be in the high end of the probability density distribution (Additional file 6; Section 1). The same is true for the Tcas5.0 draft sequence assembly N50 and percent of N50 improvement after super scaffolding compared to the other 17 of 19 total projects that had draft sequence genomes (Additional file 6: Section 1). However, in no case was the T. castaneum value the highest value recorded, suggesting that a wide range of output quality is possible including values better and worse than the output for T. castaneum.

We checked for evidence of correlations between a range of genomic metrics and map assembly, alignment or FASTA super scaffolding results. Because many of the genomic metrics had very broad ranges with variance that increased often for higher values the genomic metrics 
were $\log$ transformed to compress the upper tails and stretch the lower tails of the distributions.

Overall we found little correlation between either sequence FASTA N50, molecule map coverage or molecule map label density and final genome map N50. We did, however, find correlations between finished map and sequence assembly metrics and alignment and super scaffolding quality. There is a positive correlation between high value sequence assembly metrics and in silico map-to-genome map alignment metrics (Additional file 6: Sections 3-5) as well as post super scaffolding N50 improvement (Additional file 6: Sections 3,5). There is also a positive correlation between high value genome map assembly metrics and post super scaffolding N50 improvement (Additional file 6: Sections 3,5). However, no direct correlation was found between sequence assembly N50 and genome map N50 (Additional file 6: Sections 4-5). Taken together the analysis suggests different factors may determine sequence assembly and genome map assembly quality. Although sequence assembly N50 may not be useful to predict genome map N50, if both independent assemblies have high N50's than more of the map lengths may align and super scaffolding may be more productive.

The low degree of correlation found between genome map N50 and sequence N50 may stem from steps unique to the molecule map imaging process. It might be expected that a genome with sequence that assembles well may have qualities that would also favor molecule map assembly (e.g. low repeat content, low ploidy, inbreed lines, etc.). However molecule map assembly is also influenced by unique factors like frequency of fragile sites (two labels occurring on opposite strands in close proximity), labeling efficiency and ability to extract high molecular weight DNA all of which vary for different organisms.

Principal component analysis (PCA) suggests a negative correlation between labels per $100 \mathrm{~kb}$ and molecule coverage (Additional file 6: Sections 2-3). The correlation between labels per $100 \mathrm{~kb}$ and molecule coverage was weakly significant in individual regression (Additional file 6: Sections 4-5). Labels per $100 \mathrm{~kb}$ are monitored as molecules are being imaged. Lower than expected label density can occasionally lead to further labeling reactions or other adjustments to data collection and therefore greater depth of coverage.

Overall, comparison of the results for the T. castaneum genome and 19 additional genome projects suggest that results may vary widely from project to project. Many factors may contribute to this effect including the quality of the sequence assembly, degree of divergence between the organism or organisms used to extract DNA, success of extraction and labeling of high molecular weight DNA, genome size and genome complexity. In fact, the tendency for assemblies from the same genera or species to cluster together on the PCA plots suggests that organism-specific qualities may influence assembly, alignment or super scaffolding results (Additional file 6: Sections 2-3). Although analysis of more projects is needed to determine if these similarities are meaningful predictors of output quality.

\section{Conclusions}

We introduced new tools to facilitate single molecule map assembly optimization and genome finishing steps using the resultant consensus genome maps. These tools were validated using the medium-sized (200 Mb) T. castaneum beetle genome. The Tcas 3.0 genome was assembled using the gold-standard Sanger assembly strategy [15]. The Tcas 5.0 assembly benefitted from the use of LongDistance Illumina Jump libraries to anchor additional scaffolds and fill gaps. Despite this, we were able to more than triple the scaffold N50 by leveraging the optimal consensus genome maps and Stitch. We demonstrated that the AssembleIrysCluster method of optimization and Stitch can be used together to improve the contiguity of a draft genome.

As the variety of genome assembly projects increases, we are discovering that tools appropriate for all projects (e.g. genomes of varying size and complexity, assemblies of varying quality, various taxonomic groups, etc.) do not exist. Indeed, the results of Assemblation2 indicate that no one suite of datatypes or assembly workflow may be sufficient to best assemble even the subcategory of vertebrate genomes [3]. Here we described two software tools and many shorter scripts to summarize and work with these new data formats. However, we anticipate the development of a variety of bioinformatics tools for extremely long, single molecule map data as more applications for these maps are explored.

Some draft assemblies may currently be too fragmented to align to genome maps assembled from single molecule maps. However as NGS genome assemblies improve from longer read advancements existing genome maps may become useful for scaffolding new or updated sequence assemblies.

Regions where consensus genome maps disagree with sequence assemblies (e.g. negative gap lengths or partial alignments) are flagged by Stitch for investigation at a sequence level. Bioinformatics tools that could automate assembly editing based on such discrepancies are needed to fully support genome improvement with consensus genome maps.

\section{Availability and requirements \\ Pipelines and tutorials}

Project name: Sewing machine pipeline

Project home page: The Sewing machine script and tutorial are available at https://github.com/i5K-KINBRE- 
script-share/Irys-scaffolding/blob/master/KSU_bioinfo_ lab/stitch/sewing_machine_LAB.md.

Operating system(s): Linux (tested on CentOS 7, Gentoo and Ubuntu).

Programming language: Perl, Rscript, Bash

License: Pipeline script and tutorial are available free of charge to academic and non-profit institutions.

Any restrictions to use by non-academics: Please contact authors for commercial use.

Dependencies: Sewing machine requires BioPerl and BNGCompare. RefAligner is also required between iterations and can be provided by request by Bionano Genomics http://www.bionanogenomics.com/.

Project name: "Raw data-to-finished assembly and assembly analysis" pipeline

Project home page: The pipeline script and tutorial are available at https://github.com/i5K-KINBRE-scriptshare/Irys-scaffolding/blob/master/KSU_bioinfo_lab/ assemble_XeonPhi/assemble_XeonPhi_LAB.md.

Operating system(s): Xeon Phi server with 1488 threads $(6 \times 60 \times 4$ Xeon Phi co-processor threads $+24 \times 2$ Xeon host threads) and $256 \mathrm{~GB}$ of host RAM $+6 \times 8 \mathrm{~GB}$ Xeon Phi Ram, and Linux CentOS 7.

Programming language: Perl, Rscript, Bash

License: Pipeline script and tutorial are available free of charge to academic and non-profit institutions.

Any restrictions to use by non-academics: Please contact authors for commercial use.

Dependencies: AssembleIrysXeonPhi.pl and AssembleIrysCluster.pl requires DRMAA job submission libraries. RefAligner and Assembler are also required and can be provided by request by Bionano Genomics http://www.bionanogenomics.com/.

Project name: "Raw data-to-finished de novo assembly and assembly analysis" pipeline

Project home page: The pipeline script and tutorial are available at https://github.com/i5K-KINBRE-scriptshare/Irys-scaffolding/blob/master/KSU_bioinfo_lab/ assemble_XeonPhi/assemble_XeonPhi_de_novo_LAB. md.

Operating system(s): Xeon Phi server with 1488 threads $(6 \times 60 \times 4$ Xeon Phi co-processor threads $+24 \times 2$ Xeon host threads) and $256 \mathrm{~GB}$ of host RAM $+6 \times 8$ GB Xeon Phi Ram, and Linux CentOS 7.

Programming language: Perl, Rscript, Bash

License: Pipeline script and tutorial are available free of charge to academic and non-profit institutions.

Any restrictions to use by non-academics: Please contact authors for commercial use.

Dependencies: AssembleIrysXeonPhi.pl and AssembleIrysCluster.pl requires DRMAA job submission libraries. RefAligner and Assembler are also required and can be provided by request by Bionano Genomics http://www.bionanogenomics.com/.

\section{Assembly scripts}

Project name: AssembleIrysXeonPhi.pl/AssembleIrysCluster.pl

Project home page: AssembleIrysXeonPhi scripts are available at https://github.com/i5K-KINBRE-scriptshare/Irys-scaffolding/blob/master/KSU_bioinfo_lab/ assemble_XeonPhi/AssembleIrysXeonPhi.pl. The currently unsupported AssembleIrysCluster scripts are available on Github at https://github.com/i5K-KINBREscript-share/Irys-scaffolding/tree/master/KSU_bioinfo_ lab/assemble_SGE_cluster

Operating system(s): Xeon Phi server with 1488 threads $(6 \times 60 \times 4$ Xeon Phi co-processor threads $+24 \times 2$ Xeon host threads) and $256 \mathrm{~GB}$ of host RAM $+6 \times 8$ GB Xeon Phi Ram, and Linux CentOS 7 and SGE Linux (tested on a Gentoo) cluster respectively

Programming language: Perl, Rscript, Bash

License: AssembleIrysXeonPhi and AssembleIrysCluster.pl is available free of charge to academic and non-profit institutions.

Any restrictions to use by non-academics: Please contact authors for commercial use.

Dependencies: AssembleIrysXeonPhi.pl and AssembleIrysCluster.pl requires DRMAA job submission libraries. RefAligner and Assembler are also required and can be provided by request by Bionano Genomics http:// www.bionanogenomics.com/.

\section{Super scaffolding scripts}

Project name: stitch.pl

Project home page: Stitch scripts are available on Github at https://github.com/i5K-KINBRE-script-share/ Irys-scaffolding/tree/master/KSU_bioinfo_lab/stitch

Operating system(s): MAC and LINUX (tested on Gentoo and Ubuntu)

Programming language: Perl, Rscript, Bash

License: stitch.pl is available free of charge to academic and non-profit institutions.

Any restrictions to use by non-academics: Please contact authors for commercial use.

Dependencies: stitch.pl requires BioPerl. RefAligner and Assembler are also required between iterations and can be provided by request by Bionano Genomics http://www. bionanogenomics.com/.

\section{Map summary scripts}

Project name: BNGCompare.pl, bnx_stats.pl, cmap_stats.pl and xmap_stats.pl

Project home page: all scripts are available on Github at https://github.com/i5K-KINBRE-script-share/Irys-scaffol ding/tree/master/KSU_bioinfo_lab/map_tools and https: //github.com/i5K-KINBRE-script-share/BNGCompare Operating system(s): MAC and LINUX (tested on Gentoo and Ubuntu) 
Programming language: Perl, Rscript, Bash

License: bnx_stats.pl, cmap_stats.pl and xmap_stats.pl are available free of charge to academic and non-profit institutions.

Any restrictions to use by non-academics: Please contact authors for commercial use.

Dependencies: bnx_stats.pl, cmap_stats.pl and xmap_stats.pl have no dependencies.

\section{Additional files}

\section{Additional file 1: Single molecule map stretch per scan in recent} flowcells. Bases per pixel (bpp) is plotted for scans $1 . . n$ for each flowcell of mouse lemur molecules (purple). The first scan of each flowcell is indicated with a grey dashed line. The pre-adjusted molecule map stretch was determined by aligning molecule maps to the in silico maps. Data made available by P.A. Larsen, J. Rogers, A.D. Yoder and the Duke Lemur Center. (ZIP $55 \mathrm{~kb})$

Additional file 2: Cumulative length and number of single molecule maps per BNX file for T. castaneum data generated over time. Detailed metrics for molecule maps per BNX file (cumulative length and number of maps). Columns include cumulative length of molecule maps $>150 \mathrm{~kb}$, number of molecule maps $>150 \mathrm{~kb}$ and date that BNX file was generated. (CSV $4.11 \mathrm{~kb}$ )

Additional file 3: Single molecule map metrics and histograms from T. castaneum DNA. Detailed metrics for molecule maps including map N50, cumulative length and number of maps. Figures show histograms of per molecule map quality metrics including length, molecule map SNR and intensity, label count, label SNR and label intensity. Molecule maps are filter for minimum molecule lengths of 100,150 or $180 \mathrm{~kb}$. (ZIP $39.8 \mathrm{~kb}$ )

Additional file 4: Assembly of $T$. castaneum consensus genome maps with range of parameters. Detailed assembly metrics for assembled consensus genome maps using strict, default and relaxed "-T" parameter, p-value threshold are named Relaxed-T, Default-T and Strict-T respectively. The best "-T" parameter was used for two additional assemblies with either relaxed minimum molecule map length (relaxed-minlen) of $100 \mathrm{~kb}$, rather than the $150 \mathrm{~kb}$ default, or a strict minimum molecule map length (strict-minlen) of $180 \mathrm{~kb}$. (CSV $389 \mathrm{~kb}$ )

Additional file 5: ChLGs before and after super scaffolding. Alignments of Tcas5.0 and Tcas5.2 in silico maps to consensus genome maps for all ChLGs. Consensus genome maps (blue with molecule coverage shown in dark blue) aligned to the in silico maps (green with contigs overlaid as translucent colored squares). Alignment to both Tcas5.2 super scaffolds (top alignment) and Tcas5.0 scaffolds (bottom alignment) are shown. (PDF $408 \mathrm{~kb})$

Additional file 6: Assembly and super scaffolding with multiple genera. We examined experiments from 16 different genera to determine if the results seen for the Tribolium castaneum genome are typical for other genomes as well. (PDF $876 \mathrm{~kb}$ )

\section{Abbreviations}

NGS: Next-generation sequencing; BAC: Bacterial artificial chromosome; QC: Quality control; PAT: Percent aligned threshold; ChLG: Chromosome linkage groups; PCA: principal component analysis.

\section{Competing interests}

The JMS, MCC, NH, NL, and SJB declare that they have no competing interests. ETL, PS and TA are employees at BioNano Genomics and hold stock options.

\section{Authors' contributions}

MCC isolated the high molecular weight DNA and generated the image files on the Irys. ETL and JMS developed the assembly workflow. JMS wrote most of the code in the IrysScaffolding Github Repo (Stitch, AssemblelrysXeonPhi, AssemblelrysCluster, etc.). NH assisted with initial code review of analyze_irys_output (precursor to Stitch) and prepared Tcas5.0. JMS and NL manually edited Tcas5.1. JMS performed the data analyses. TA contributed to sections discussing BioNano RefAligner and Assembler. PS contributed to interpretation of results. JMS and SJB did most of the writing with contributions from all authors. All authors read and approved the final manuscript.

\section{Acknowledgements}

This project was supported by an Institutional Development Award (IDeA) from the National Institute of General Medical Sciences of the National Institutes of Health under grant number P20 GM103418. The content is solely the responsibility of the authors and does not necessarily represent the official views of the National Institute of General Medical Sciences or the National Institutes of Health.

Data for Additional file 1 and Additional file 6 was kindly made available by P.A. Larsen, J. Rogers, A.D. Yoder and the Duke Lemur Center.

The Tribolium castaneum genome project is part of the i5k Genome Sequencing Initiative for Insects and Other Arthropods.

Matthias Weissensteiner \& Jochen Wolf, Uppsala University. Stephen Schaeffer from The Pennsylvania State University and Stephen Richards from the Baylor College of Medicine Human Genome Sequencing Center for the use of the D. pseudoobscura data. Mike Kanost from Kansas State University. Jeff Maughan from Brigham Young University for the use of the Amaranth data. The Udall Lab from Brigham Young University and Cotton Inc. for the use of the cotton data. Grant (NSF 1237993) for use of the Medicago data. Christopher Cunningham, University of Georgia for the use of Nicrophorus data. Catherine Peichel from the Fred Hutchinson Cancer Research Center and Michael White from the University of Georgia for the Gasterosteus data. Mirkó Palla, Ph.D., Wyss Institute Postdoctoral Fellow, Church; Laboratory - Department of Genetics, Harvard Medical School and George Church, Ph.D., Wyss Institute Core Faculty Member, Robert Winthrop Professor of Genetics at Harvard Medical School, Professor of Health Sciences and Technology at Harvard and MIT, and Senior Associate Member at the Broad Institute of Harvard and MIT for the Escherichia coli data.

\section{Author details}

${ }^{1}$ KSU/K-INBRE Bioinformatics Center, Division of Biology, Kansas State University, Manhattan, KS, USA. ${ }^{2}$ BioNano Genomics, San Diego, CA, USA.

Received: 20 April 2015 Accepted: 9 September 2015

Published online: 29 September 2015

\section{References}

1. Salzberg SL, Phillippy AM, Zimin A, Puiu D, Magoc T, Koren S, et al. Gage: A critical evaluation of genome assemblies and assembly algorithms. Genome Res. 2012;22(6):. doi:10.1101/gr.131383.111.

2. Vezzi F, Narzisi G, Mishra B. Feature-by-feature-evaluating de novo sequence assembly. PLoS ONE. 2012;7(2):. doi:10.1371/journal.pone. 0031002.

3. Bradnam K, Faas J, Alexandrov A, Baranay P, Bechner M, Birol I, et al. Assemblathon 2: evaluating de novo methods of genome assembly in three vertebrate species. GigaScience. 2013;2(10):. doi:10.1186/2047217X-2-10.

4. Das SK, Austin MD, Akana MC, Deshpande P, Cao H, Xiao M. Single molecule linear analysis of dna in nano-channel labeled with sequence specific fluorescent probes. Nucleic Acids Res. 2010;38(e177): doi:10.1093/nar/gkq673.

5. PacBio P6-C4 Chemistry Specifications. http://investor.pacificbiosciences. $\mathrm{com} /$ releasedetail.cfm?ReleaselD $=876252$.

6. Illumina Long Distance Jump Library Specifications. http://www.operon. com/services/next-generation-sequencing/illumina/libraries.aspx.

7. Quick J, Quinlan AR, J L. N. A reference bacterial genome dataset generated on the minion portable single-molecule nanopore sequencer. GigaScience. 2014;3(22): doi:10.1186/2047-217X-3-22.

8. McCoy RC, Taylor RW, Blauwkamp TA, Kelley JL, Kertesz M, Pushkarev D, Petrov DA, A F.-L. Illumina truseq synthetic long-reads empower de novo assembly and resolve complex, highly-repetitive transposable elements. PLOS ONE. 2014;9(9):. doi:10.1371/journal.pone.0106689.

9. Teague B, Waterman MS, Goldstein S, Potamousis K, Zhou S, Reslewic S, et al. High-resolution human genome structure by single-molecule analysis. PNAS. 2012;107(24):: doi:10.1073/pnas.0914638107.

10. Opgen Argus MapCard Specifications. http://www.tgac.ac.uk/ sequencing-platforms/. 
11. BioNano IrysChips Specifications. http://www.bionanogenomics.com/ wp-content/uploads/2015/01/datasheet-web.pdf.

12. Cao H, Hastie AR, Cao D, Lam ET, Sun Y, Huang H, et al. Rapid detection of structural variation in a human genome using nanochannel-based genome mapping technology. GigaScience. 2014;3(34): doi:10.1186/2047-217X-3-34.

13. Ross MG, Russ C, Costello M, Hollinger A, Lennon NJ, Hegarty R, et al. Characterizing and measuring bias in sequence data. Genome Biol. 2013;14(R51):. doi:10.1186/gb-2013-14-5-r51.

14. Mendelowitz L, Pop M. Computational methods for optical mapping. GigaScience. 2014;3(33):. doi:10.1186/2047-217X-3-33.

15. Richards S, Gibbs RA, Weinstock GM, Brown SJ, Denell R, Beeman RW, et al. The genome of the model beetle and pest tribolium castaneum. Nature. 2008:452:949-55. http://dx.doi.org/10.1038/nature06784.

16. Lorenzen MD, Doyungan Z, Savard J, Snow K, Crumly LR, Shippy TD, et al. Genetic linkage maps of the red flour beetle, tribolium castaneum, based on bacterial artificial chromosomes and expressed sequence tags Genetics. 2005;170(2)::741-7. doi:10.1534/genetics.104.032227.

17. Lam ET, Hastie A, Lin C, Ehrlich D, Das SK, Austin MD, et al. Genome mapping on nanochannel arrays for structural variation analysis andsequence assembly. Nat Biotechnol. 2012;30(8):. doi:10.1038/nbt.2303.

18. Anantharaman T, Mishra B. A probabilistic analysis of false positives in optical map alignment and validation; 2001. https://cs.nyu.edu/mishra/ PUBLICATIONS/01.falsepos.ps.

\section{Submit your next manuscript to BioMed Central} and take full advantage of:

- Convenient online submission

- Thorough peer review

- No space constraints or color figure charges

- Immediate publication on acceptance

- Inclusion in PubMed, CAS, Scopus and Google Scholar

- Research which is freely available for redistribution

Submit your manuscript at www.biomedcentral.com/submit 